\title{
NEUTRALIZING CYLINDER GLASSES AS TEST FOR MALINGERING
}

$\mathrm{BY}$

\author{
H. Lytton, \\ EALING
}

IT is not easy to outwit a malingerer claiming a reduction of his visual acuity to approximately the same extent in both eyes. Some suspicion is aroused if the examinee reads fluently the first three or four lines of the sight-test and suddenly stops, as if he has decided beforehand how far down he will read. A clever malingerer, who has a certain knowledge of the principles underlying our tests, will not commit this mistake, nor will he be trapped by the increase of the subtended visual angle at a closer range. Prism tests and other methods, which may demonstrate a condition of binocular vision, do not exclude the reduction of the sight of both eyes to nearly the same degree.

In such cases, I have found the following test useful in finding out if the suspicion of malingering is justified. In addition to the glass, which gives the best possible correction, a +cylinder glass, e.g., + cyl. 1.0 axis $90^{\circ}$ is put in the posterior immobile compartment of the trial frame and the corresponding - cylinder glass - cyl. 1.0 axis $45^{\circ}$ is placed in the revolving compartment in front. Now, the examinee is asked to change the axis of the -cylinder by turning the adjusting screw until he has got the clearest definition of the test types. It speaks strongly for his bona fides if he turns the axis into the neutralising position of 90 degrees. In the absence of any visible cause careful colour scotometry may supply an explanation of the case.

A malingerer is likely to put the axis at 180 degrees.- If he still claims to see the fourth line of the sight test indicating the visual acuity $6 / 18$, it is proved that his vision is at least $6 / 9$, as the crossed cylinder reduces the visual acuity to about 50 per cent.

\section{ANNOTATION \\ The Biter Bitten}

The following story bears a moral and may be of interest. About twenty years ago a gentleman was referred to the writer for glasses. The patient was a man of about 62 years of age and had never worn any correction. He started life as a medical student, but found the work distasteful and went into business instead. He did well and when we first knew him he was retired and living in the country. His ophthalmic condition was perfectly straightforward. He had 